\title{
Artifacts Removal from ECG Signal Using a Multistage MNLMS Adaptive Algorithm
}

\author{
Aamir Mehmood $^{1 *}$, Muhammad Iram Baig ${ }^{2}$, Ehtasham-ul-Haq ${ }^{3}$ and Laeeq Aslam ${ }^{4}$ \\ ${ }^{1,2,3,4}$ Department of Computer Engineering, University of Engineering \& \\ Technology, Taxila Pakistan \\ 1aamir_mehmood19@yahoo.com, ${ }^{3}$ ehatishamuet@gmail.com
}

\begin{abstract}
Electrocardiogram (ECG) signal is the representation of heart's electrical potential. ECG signal is a small amplitude signal and usually contain numerous types of noises which are classified on the basis of their frequency content. Noises that mostly corrupt the ECG signals are Power line interference, Instrumentation noise, External electromagnetic interference, Baseline drift and noise due to the random movement of the body and respirational movement. The removal of such types of noises is very essential for better analysis of ECG signals, which results in a better estimation of the human cardiac system. Many algorithms have been developed for the removal of these artifacts from the ECG signals to extract required information. The primary method is to pass the signal containing the noise through fixed or static filters such as high pass, low pass or band pass filters depending upon the nature of the noise. The static filters have fixed filter coefficients which makes it difficult to remove time varying noise from the signals. To overcome this shortcoming of the fixed filters, different adaptive filtering methods have been developed. Since the ECG signal suffers from several artifacts at a time, which makes a single stage adaptive filter unsuitable for multiple noise signals removal. This paper presents a Multistage Modified Normalized Least Mean Square (MNLMS) algorithm for the removal of multiple artifacts from ECG signals. The results of the proposed algorithm are compared with existing adaptive algorithms including Multistage LMS, Multistage NLMS, Multistage RLS, Multistage SDLMS, on the basis of metrics, including Signal to Noise Ratio (SNR), convergence rate and the computational time, which demonstrate the effectiveness of the proposed algorithm.
\end{abstract}

Keywords: ECG, Noise Removal, Multistage Filtering, Adaptive algorithms

\section{Introduction}

The ECG signals represent the electrical activity of the heart. The ECG signal is recorded by a noninvasive technique; in this technique the electrodes are placed at several predefined points on the human body. These signals provide information regarding the performance of the heart and associated cardiac diseases and can be used in expert systems for recommending surgical procedures, etc. The ECG signal and heart rate determines the cardiac health of the human heart. Any disorder in the heart rate or rhythm or change in the morphological features of the ECG wave (PQRST) is an indication of cardiac arrhythmia [2].

The amplitude and duration of the features of ECG wave contain useful information about the performance and type of diseases related to the heart. So correct detection of features of the ECG wave is an important field of research. The amplitude of ECG Signal or other physiological signals is very low typically of 1 to $2 \mathrm{mv}$ and requires a bandwidth of 0.1 to $120 \mathrm{~Hz}$, so a small external interference (environment noise) may greatly affects

Received (June 10, 2017), Review Result (November 6, 2017), Accepted (November 13, 2017) 
the signal quality. During data acquisition and transmission, the ECG wave suffers from many artifacts which corrupt the true information bearing signal. The signal becomes noisy and is not useful for clinical diagnosis since it contains certain artifacts like motion induced artifacts, muscle induced artifacts, power line interference, instrumentation noise and noise due to the random movement of the body known as electromyography (EMG). This artifact greatly degrades the signal integrity, frequency resolution and strongly affects the morphological features of the signal containing information. It is very important to reduce or remove these artifacts and accurately extract the important parameters of the ECG signal for reliability and better clinical diagnosis.

Many algorithms have been developed to remove these artifacts and extract the information from the ECG, like analog devices, electronic circuits and digital filters. As the computational power of microprocessor increases, the noise removal problems have been mostly shifted to the digital filters because of their flexibility, efficiency, less memory storage and accuracy. The primary method is to pass the signal containing the noise through a high pass, low pass or band pass filter depending upon the nature of the noise; these filters are known as fixed or static filters. The static filters have fixed filter coefficients so it is difficult to remove the time varying noise like electromyogram (EMG) or Instrumentation noise. To overcome this shortcoming of the fixed filters, different adaptive filtering methods have been developed.

The adaptive filtering is the most popular technique for the de-noising of ECG signal amongst all due to the nonlinear nature of the ECG signal. In [1], the authors evaluated the performance of LMS and RLS adaptive algorithm and found that the RLS based adaptive filters have better performance. In [3], have used several simple and efficient sign based normalized adaptive filters for the cancellation of artifacts in ECG, which are computationally superior having multipier free for update loops. The authors in [4], compared the performance of adaptive filter with and without reference noise, adaptive filter without reference noise has better performance since the noise in the primary signal may or may not correlate with the reference input to the adaptive filter.

The work presented in [5], discusses a Novel Modified LMS algorithm for the removal of PLI noise. According to the paper, the algorithm was less complex and more accurate than the existing adaptive filtering algorithm. The work presented in [6], proposed a modified normalized least mean square algorithm (MNLMS) for removing the noise generated by power line interferences and achieved much better results in term of signal to noise ratio, convergence rate and computational complexity as compared to the recently published literature. However, in practical applications beside PLI the ECG contains other types of noise such as baseline wander and EMG noise. Therefore, the MNLMS algorithm proposed in [6], is only suitable for a particular scenario where an ECG signal is corrupted by PLI noise.

In this research work, we present an algorithm for removing multiple noise signals, i.e., baseline wander, EMG and PLI noise signals from an ECG signal using a multistage MNLMS algorithm. This makes the proposed algorithm more robust against noisy environments and suitable for practical application point of view. Moreover, the proposed algorithm is compared with existing multistage LMS, NLMS, SDLMS and RLS algorithms on the basis of performance metrics, i.e., SNR, computational time and the convergence rate. The results for the proposed algorithms are found better than existing algorithms in term of the SNR and rate of convergence.

\section{Multistage Adaptive Filtering}

Adaptive filter is employed for modeling the behavior of non-linear signals which vary in time such as echo cancellation, system recognition, or signal improvement [11]. The coefficients of adaptive filter change according to environmental changes that may occur due to the time varying property of the signals [7]. 
Since the ECG signal suffers from several artifacts like Power Line Interference PLI, Electromyogram (EMG). So a single stage adaptive filter will not be able to eliminate all the artifacts existing in the ECG signals because an adaptive filter works with only one reference input signal that correlates to one type of the noises present in the ECG signals. Therefore, to overcome this issue, a multistage adaptive filtering approach has been proposed having three reference inputs correlated to three different types of artifacts present in the ECG signal. All the adaptive algorithms discussed in this paper are used as Multistage Adaptive Filters. The structure of the adaptive filter is shown below. The literature review gives some prior knowledge about the amplitudes and the frequencies of the noise artifacts present in the ECG signal. The generated reference signals correlate with different types of the noise.

An error signal updates the weights of the adaptive filter algorithm. In this research work, we have proposed multistage adaptive filter that operates in two stages. In the first stage, it eliminates the electromyogram artifacts and the baseline wander noise as shown in Figure 1. The output of the first stage filtering is coupled to the second stage filtering where the power line interference noise is eliminated and a noise-free signal is attained at the output as shown in Figure 2.

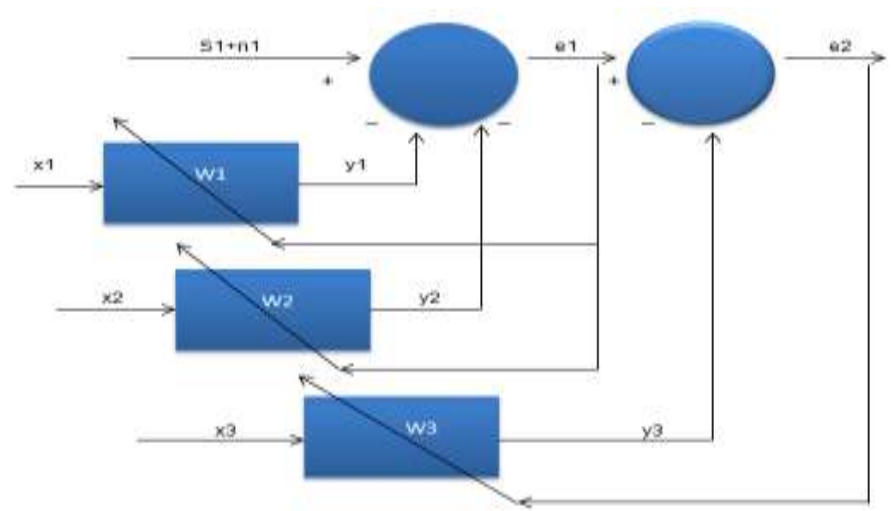

Figure 1. Multistage Adaptive Filtering

The reference signal is used as an input to the adaptive filter. The received signal is the mixture of the primary input signal and noise signal as shown in Figure 1.

$$
r(t)=\text { signal }+ \text { noise }
$$

The output of the filter

$$
y(m)=W^{T}(m) * u(m)
$$

Where $W^{T}(m)$ represents the initial filter coefficients/weights and $u(m)$ is the reference signal. An error signal is attained by subtracting the output of the filter from the received signal as shown in Figure 1.

$$
e(m)=s(m)-y(m)
$$

The Error Signal e $(\mathrm{m})$ is used as a feedback to the adaptive filter and the process runs iteratively till the error signal is removed.

\subsection{Least Mean Square Algorithm (LMS)}

LMS is a well-known stochastic gradient based algorithm that is used to solve nonlinear type problems because of its simplicity and less complexity, because of this it can be implemented with finite precision arithmetic. It is a search based procedure in 
which interpretation of the gradient vector is completed by minimizing the objective function.

The formula for updating the weights for LMS filter is given as follows [8].

$$
W(m+1)=W(m)+2 * \mu * x(m) * e(m)
$$

$w(m+1)$ is filter weight, which is updated, $w(m)$ is the filter's current weight, $e(m)$ is the error signal, $x(m)$ represents the reference input signal and $\mu$ shows the step size which is an important parameter in determining the convergence and stability of the LMS filter.

\subsection{Normalized Least Mean Square Algorithm (NLMS)}

The major problem in LMS algorithm was the selection of the step size value as the convergence rate and the filter performance was dependent on this parameter. Typically, a small step size is chosen in LMS algorithm to achieve the optimal coefficient compromising on the convergence of the filter.

To overcome the issues of LMS filter, NLMS filter is employed. In the NLMS algorithm, the step size is normalized based on normalizing the power of input and thus the stability of the filter is maintained.

The equation for adapted weights of the NLMS filter is as follows [9].

$$
W(m+1)=W(m)+2 * \frac{\mu}{x(m) x^{T}(m)} * x(m) * \mathrm{e}(\mathrm{m})
$$

$W(m+1)$ represents the updated weight, $W(m)$ represents the initial weight, $\mathrm{x}(\mathrm{m})$ is the input signal, e $(\mathrm{m})$ shows the error signal and $\frac{\mu}{x(\mathrm{~m}) x^{T}(\mathrm{~m})}$ characterizes the normalized step size.

The convergence of NLMS algorithm is faster as compare to the LMS because of the normalized step size. In other words, if the error is minor, the step size becomes smaller and if the error is large the step size becomes large.

\subsection{Sign Data Least Mean Square Algorithm (SDLMS)}

SDLMS algorithm is obtained by replacing the input signal $x(m)$ by $\operatorname{sign}(x)$ in the conventional LMS algorithm.

$$
\operatorname{sgn}(x)=\left\{\begin{array}{rr}
1 & x>0 \\
-1 & x<0 \\
0 & x=0
\end{array}\right.
$$

SDLMS is computationally cheaper than the LMS algorithm because of quantization of the error which is done by a simple sign function for faster adaptation.

The formula for the weights updating for SDLMS is as follows [10].

$$
W(m+1)=W(m)+2 * \mu * \operatorname{sgn}\{x(m)\} * e(m)
$$

\subsection{Recursive Least Mean Square Algorithm (RLS)}

Least square Algorithm tries to minimize the sum of squares of difference between a desired signal and the filter output. When new samples arrive after every iteration, thus solution of least square can be computed in a recursive way forming a new Recursive least square (RLS) Algorithm. 
The RLS algorithm is prevalent because of its fast convergence rate and better performance in non-stationary settings.

The objective function for the RLS algorithm is given as:

$$
\begin{aligned}
& \varepsilon^{d}(n)=\sum_{i=0}^{n} \beta^{(n-i)} * \varepsilon^{2}(i) \\
& \varepsilon^{d}(n)=\sum_{i=0}^{n} \beta^{(n-i)}\left[d(i)-x^{T}(i) * w(n)\right]^{2}
\end{aligned}
$$

Where $w(n)=[w(0) w(1) w(2) \ldots . . w(m-1)]$ represtns the weight vector, $\varepsilon(i)$ is the aposteriori error at sample $\mathrm{i}$ and parameter $\beta$ is the forgetting factor. Each error is comprised of of the difference between the desired sample and the filter output using the most recently updated filter coefficents w(n) [14].

The optimal weight vector $\mathrm{w}(\mathrm{k})$ for RLS algorithm is given as:

$$
w(k)=\left[\sum_{i=0}^{k} \beta^{k-1} x(i) x^{T}(i)\right]^{-1} * \sum_{i=0}^{k} x(i) d(i)
$$

\subsection{Proposed Modified Normalized Least Mean Square Algorithm (MNLMS)}

The updated weight vector for the Modified NLMS is given by.

$$
\mathrm{w}(\mathrm{m}+1)=\mathrm{w}(\mathrm{m})+\mu /\left[\mathrm{x}(\mathrm{m}) * \mathrm{x}^{\prime}(\mathrm{m})\right] * \mathrm{Q}\{\mathrm{e}(\mathrm{m})\} * \mathrm{x}(\mathrm{m})
$$

Where Q[.] is the logarithmic quantization function which can be given as [6].

$$
\mathrm{Q}(\mathrm{u})=\alpha * 2 \theta(\mathrm{u}) * \operatorname{sign}(\mathrm{u})
$$

In this algorithm, the ECG signal processing is divided in two different stages i.e., Convergence stage and Extracting stage. In Convergence, the proposed algorithm reiterates with faster convergence rate to achieve a principal convergence. When principal convergence is attained, the algorithm goes into the extraction stage.

$$
\Theta(\mathrm{u})=\log 2 \mathrm{lu} / \alpha \mathrm{l}
$$

At convergence stage, $\alpha$ is always less than 1 and denotes the power of two values. The term $\mathrm{u} / \alpha$ is amplifies the ECG signal to integer type by performing shifting operation. When convergence is attained, the algorithm reiterates to extract required information from the signal using the following equation.

$$
\Theta(u)=-\log 2 \mathrm{lu} / \alpha \mathrm{l}
$$

At extraction stage, a threshold value $€$ is introduced. When the output of the system becomes smaller than or equal to $€$ for a large number of successive iterations that i.e., $|e(m)-e(m-1)|<€$, then the convergence state is attained and algorithm comes to the second stage.

\section{Methodology of Research}

The proposed methodology for the removal of artifacts from ECG signal is shown in Figure 2. An ECG signal, i.e., $\mathrm{x}$ (n) is transmitted through wearable wireless sensors. During the data acquisition and signal transmission, three different types of noise signals, i.e., Electromyogram (EMG), Baseline Wander (BW) and Power line interference (PLI) is mixed up with the ECG signal [4]. A Multistage Modified Normalized Least Mean 
Square (MNLMS) adaptive filter is then applied to the noisy ECG signal for the cancellation of these artifacts. The filter consists of two stages as shown in Figure 2. The first stage removes EMG and BW noises whereas the second stage removes PLI noise.

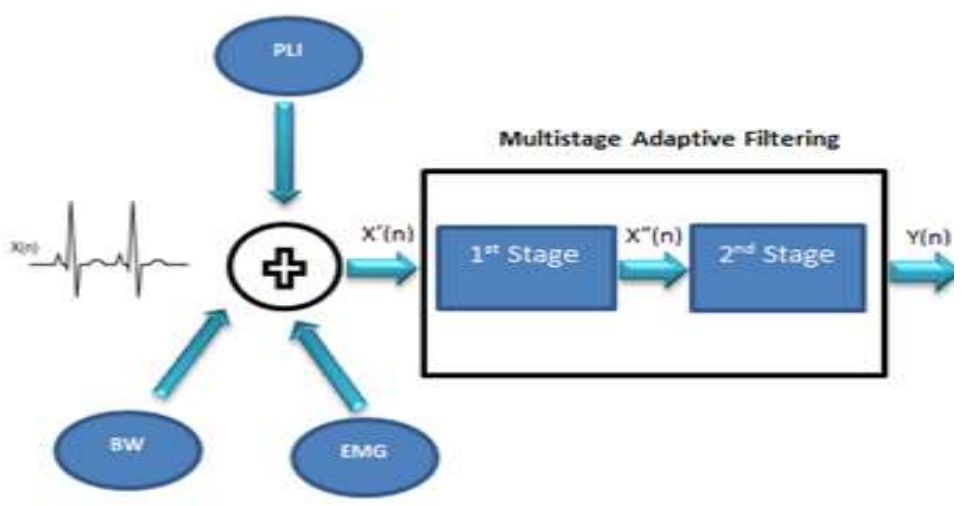

Figure 2. Proposed Methodology

\subsection{Dataset}

The data set for this research has been taken from MIT-BIH database. Massachusetts Institute of Technology and Boston Beth Hospital have established an online database for the purpose of research in arrhythmia and other related cardiac issues [14]. The data set contains 48 hours of ECG signals recordings, which are gathered from 47 subjects. About $60 \%$ of subjects belong to inpatient and $40 \%$ belongs to outpatient. Ten ECG signals are extracted from this database to validate the results.

\subsection{Implementation}

For implementation of the proposed scheme, MATLAB 2015 tool is used. The artifacts are also generated in MATLAB. The Baseline Wander noise is generated with a frequency range between 0.5 to $5 \mathrm{~Hz}$. The Power line interference (PLI) noise is generated by using a sine wave of $60 \mathrm{HZ}$. The third artifact is the motion artifact, i.e., EMG, with the frequency range of more than $100 \mathrm{~Hz}$. These three artifacts are generated in MATLAB and then added to the original signal to corrupt it. The step size for linear and nonlinear algorithms and filter order is explained in Table 1. All multistage adaptive algorithms discussed in section this are then applied to the noisy ECG signal and their performances are evaluated in terms of SNR, Convergence rate and Computational time. The results are described in details in the next section.

Table 1. Filter Parameters

\begin{tabular}{llll}
\hline Filters & $\begin{array}{l}\text { Linear Step } \\
\text { Size }\end{array}$ & $\begin{array}{l}\text { Nonlinear } \\
\text { Step Size }\end{array}$ & $\begin{array}{l}\text { Filter } \\
\text { order }\end{array}$ \\
\hline Filter 1 & 0.006 & 0.005 & 10 \\
Filter 2 & 0006 & 0.005 & 10 \\
Filter 3 & 0.004 & 0.004 & 200 \\
\hline
\end{tabular}

\section{Performance Analysis}

\subsection{Simulation Results}

In simulation, the input to multistage adaptive filters is an original ECG signal correlated with the PLI, Baseline wander and EMG type noise. The filter order for first 
stage filters i.e., filter 1 and filter 2, was chosen to be 10, linear and nonlinear step size was chosen to be 0.006 and 0.005 respectively. The filter order for second stage filter i.e., filter 3 was chosen to be 200, linear and nonlinear step size for filter 3 was chosen to be 0.004. Figures $3 \& 4$ shows the original ECG signal and ECG signal corrupted by PLI, Baseline wander and EMG type noise. Simulation results for multistage LMS, NLMS, SDLMS, RLS and MNLMS along with mean square error (MSE) are also shown.

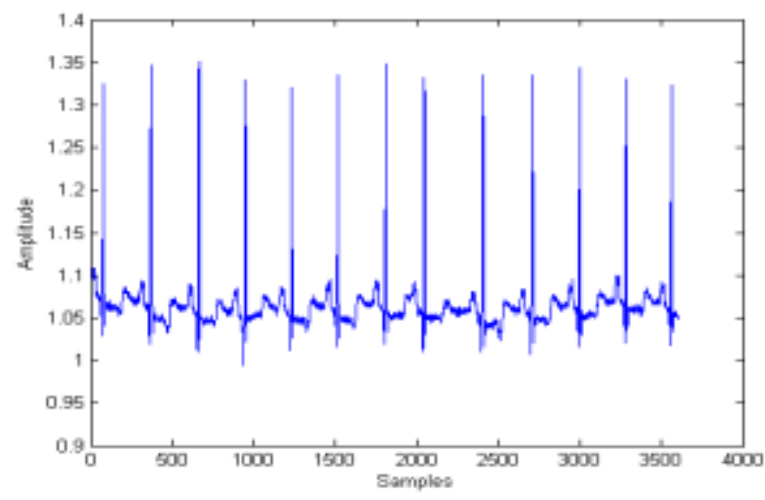

Figure 3. Original ECG Signal

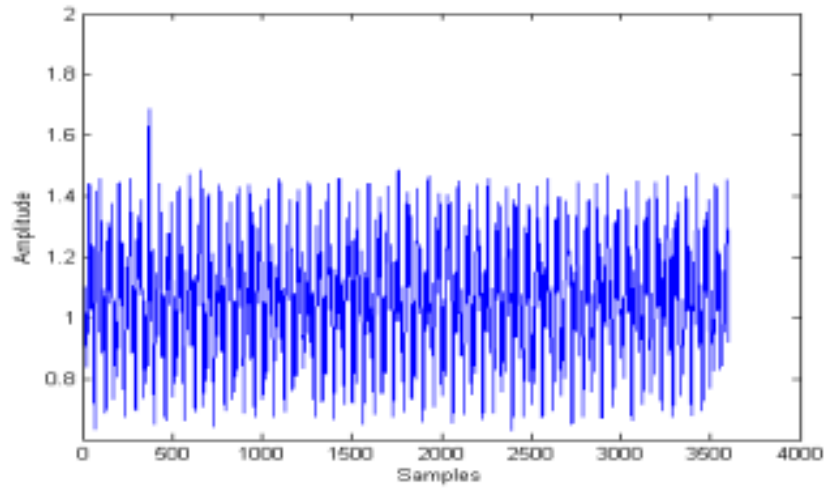

Figure 4. Noisy ECG Signal
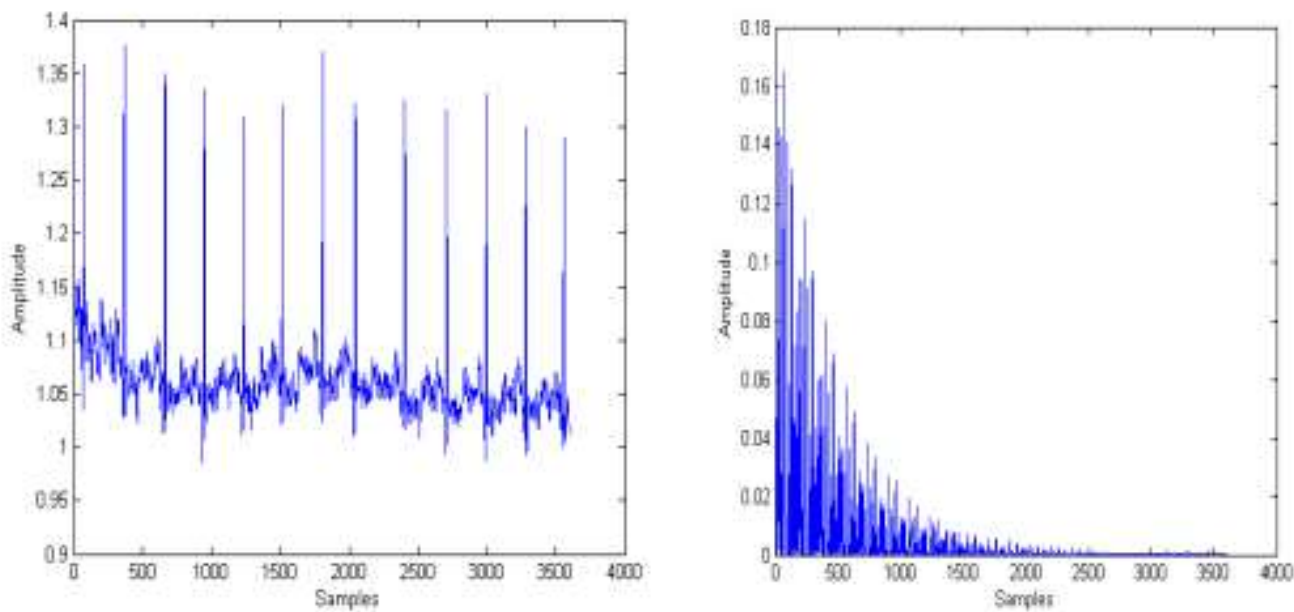

Figure 5. Filtered Signal \& MSE Plot Using LMS 

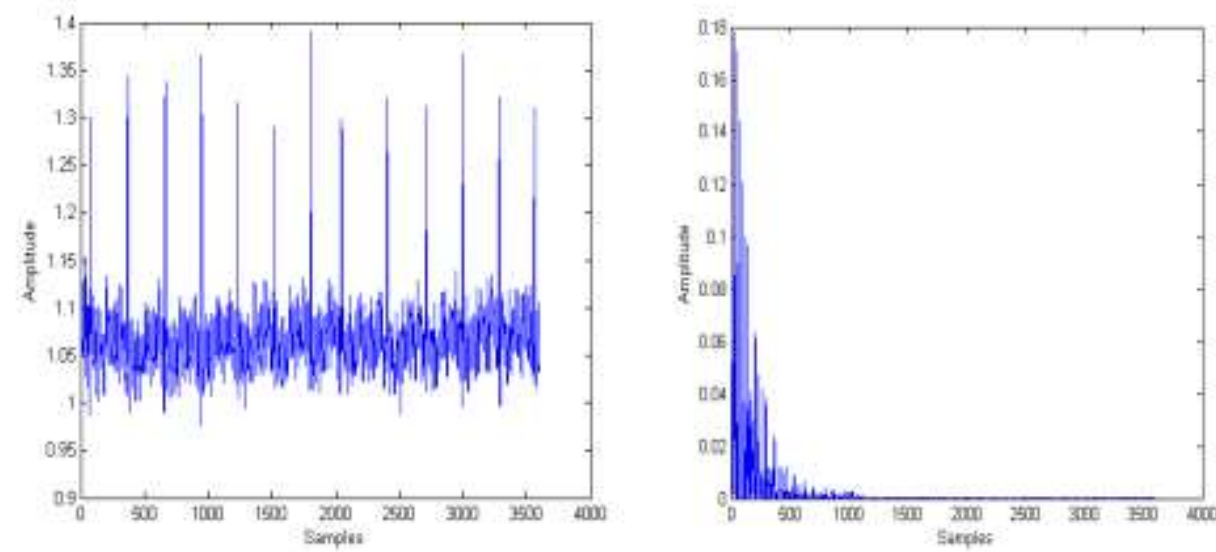

Figure 6. Filtered Signal \& MSE Plot Using NLMS
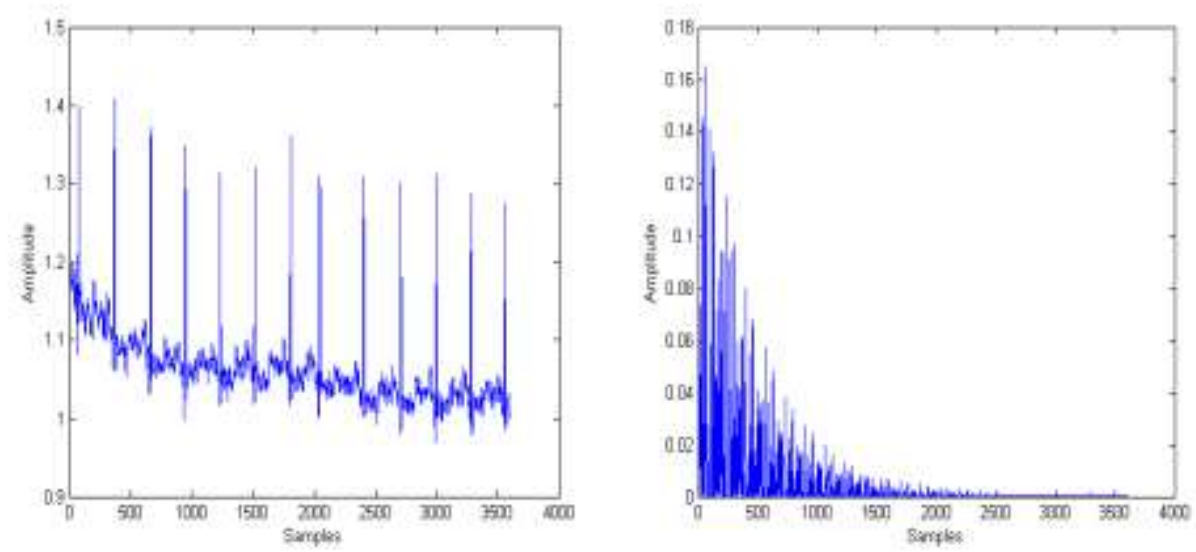

Figure 7. Filtered Signal \& MSE Plot Using SDLMS
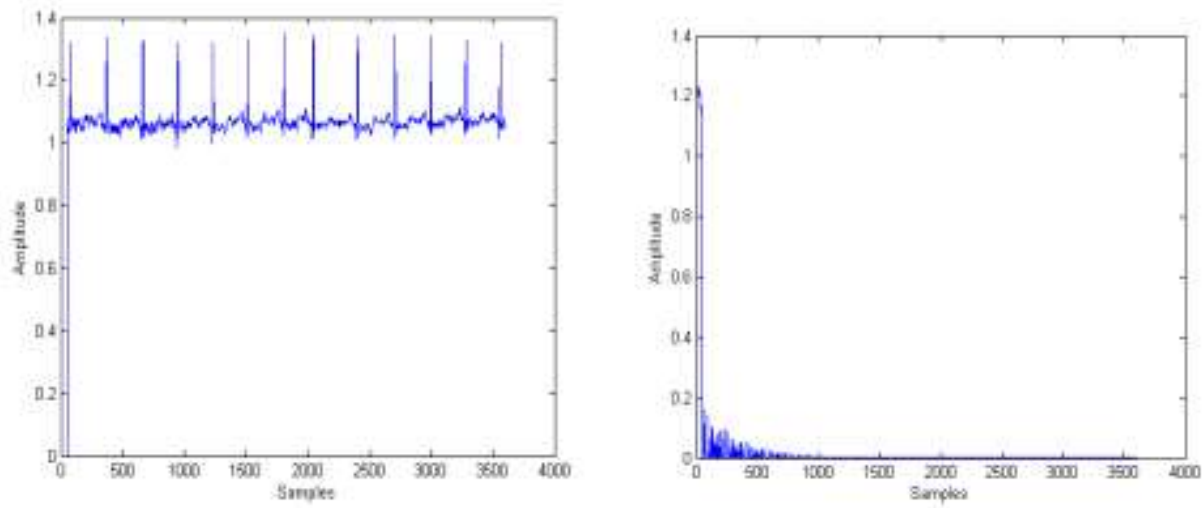

Figure 8. Filtered Signal \& MSE Plot Using RLS 

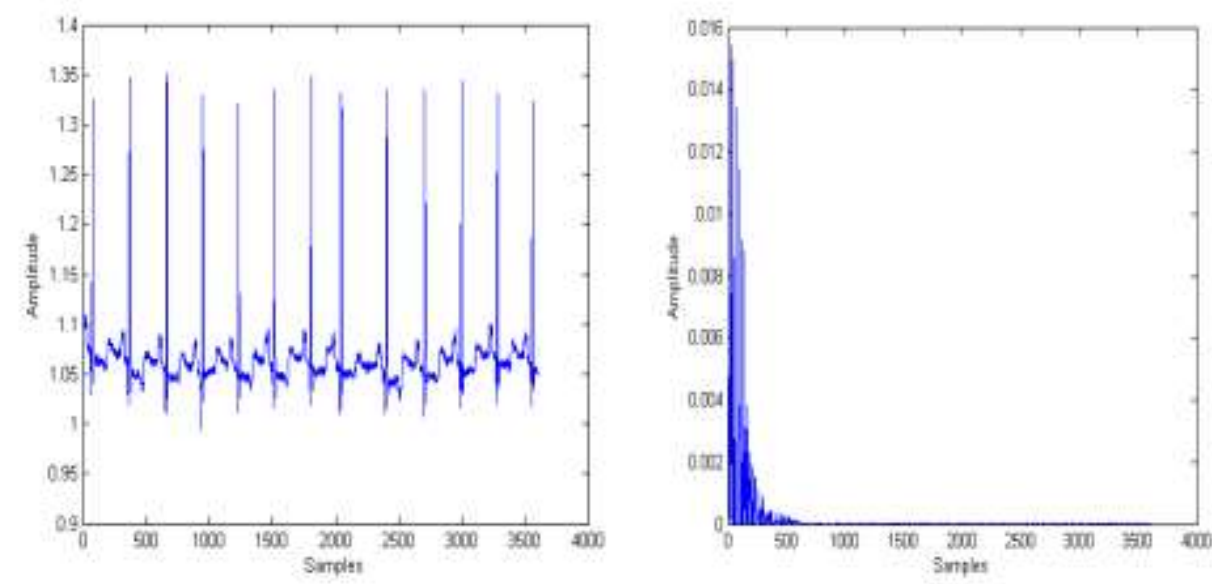

Figure 9. Filtered Signal \& MSE Plot Using MNLMS

\subsection{Discussion}

In this research work, we have analyzed the response of different multistage adaptive algorithms on multiple artifacts and a comparison is done between different multistage adaptive filters including LMS, NLMS, SDLMS, RLS and MNLMS based on performance metrics of SNR, convergence rate and time complexity. Table 2 shows the overall averaged results. Time complexity is measured in seconds and convergence rate is measured in samples. SNR is represented in dbs. It can be observed from Table 2 that the MNLMS achieves the best performance as compared to other algorithms. MNLMS gives the best SNR and convergence speed. The RLS also provides better performance. However, the computational complexity of the RLS algorithm is the highest amongst all other algorithms, as the algorithm is recursive and uses more past data for the calculation of the next coefficient.

The performance of LMS algorithm is also good but it converges very slowly. On the other hand, it is simple and less complex than RLS. NLMS achieves faster adaptation but we have to sacrifice on its performance.

Table 2. Comparison of Proposed Algorithm

\begin{tabular}{llll}
\hline Algorithm & $\begin{array}{l}\text { SNR } \\
\text { (dB) }\end{array}$ & $\begin{array}{l}\text { Convergence } \\
\text { Rate(samples) }\end{array}$ & $\begin{array}{l}\text { Computational } \\
\text { Time(seconds) }\end{array}$ \\
\hline LMS & 28.61 & 2600 & 0.44 \\
NLMS & 26.27 & 1100 & 0.57 \\
SDLMS & 23.87 & 2400 & 1.02 \\
RLS & 34.14 & 900 & 1.44 \\
MNLMS & $\mathbf{3 6 . 3 3}$ & $\mathbf{6 0 0}$ & $\mathbf{1 . 1 5}$ \\
\hline
\end{tabular}

\section{Conclusion}

The removal of noises from Electrocardiogram (ECG) signal is very essential for efficient analysis of ECG signals for better estimation of the human cardiac system. Many algorithms that have been developed for the noise removal from ECG signals have some limitation. The static filters have fixed filter coefficients which makes it difficult to remove time varying noise from the ECG signals. Also, a single stage adaptive filter is unsuitable for removing multiple noise signals from an ECG signals. This research work presents a Multistage Modified Normalized Least Mean Square (MNLMS) algorithm for the removal of multiple artifacts from ECG signals. The results of the proposed algorithm are compared with existing adaptive algorithms including Multistage LMS, Multistage 
NLMS, Multistage RLS, Multistage SDLMS, Multistage RLS, on the basis of metrics, including Signal to Noise Ratio (SNR), convergence rate and the computational time, which demonstrate the effectiveness of the proposed algorithm.

ECG is still an open and widespread area for research in signal processing. In future work, Wavelet transform can be applied for features extraction from ECG signal and distance between two consecutive $\mathrm{R}$ peaks can be measured for determining the heart rate. In automatic ECG analysis the decision has to be made by the machine so if the machine gets more purified signal it can analyze the ECG signal in a better way and the chances of error are minimized. Furthermore, the proposed approach can be implemented in an ehealth system for the better analysis of biomedical signal.

\section{Acknowledgments}

Firstly, we would like to thanks to my advisor Prof Dr. Muhammad Iram Baig for the continuous support of our research, for his patience and immense knowledge. His guidance helped me in all the time of research and writing. Second we would like to thanks to UET, Taxila for providing us opportunity to do the research.

\section{References}

[1] S. Singh, "Performance Evaluation of Different Adaptive Filters for ECG Signal Processing", (2010).

[2] M.G. Khan, "Rapid ECG interpretation", Springer Science \& Business Media, (2008).

[3] M.Z.U. Rahman, R.A. Shaik and D.R.K. Reddy, "Efficient sign based normalized adaptive filtering techniques for cancelation of artifacts in ECG signals: Application to wireless biotelemetry", Signal Processing, vol. 91, no. 2, (2011), pp.225-239.

[4] C.H. Chang, H.J. Ko and K.M. Chang, "Cancellation of high-frequency noise in ECG signals using adaptive filter without external reference", In Biomedical Engineering and Informatics (BMEI), 2010 3rd International Conference on, IEEE, vol. 2, (2010), pp. 787-790.

[5] Y. Jiao, R.Y. Cheung and M.P. Mok, "Modified Log-LMS adaptive filter with low signal distortion for biomedical applications", In Engineering in Medicine and Biology Society (EMBC), 2012 Annual International Conference of the IEEE, IEEE, (2012), pp. 5210-5213.

[6] S. Khan, S.M. Anwar, W. Abbas and R. Qureshi, "A Novel Adaptive Algorithm for Removal Of Power Line Interference From Ecg Signal”, Science International, vol. 28, no. 1, (2016).

[7] S.S. Haykin, "'Adaptive filter theory”, Pearson Education India, (2008).

[8] R. Panda, "Removal of Artifacts from Electrocardiogram (Doctoral dissertation, National Institute of Technology Rourkela)", (2012).

[9] S.A. Rehman and R.R. Kumar, "performance comparison of adaptive filter algorithms for ECG signal Enhancement", International Journal of Advanced Research in Computer and Communication Engineering, vol. 1, no. 2, (2012), pp.255-271.

[10] R. Sameni, M.B. Shamsollahi, C. Jutten and G.D. Clifford, "A nonlinear Bayesian filtering framework for ECG denoising", IEEE Transactions on Biomedical Engineering, vol. 54, no. 12, (2007), pp.21722185.

[11] S.G. Mallat, "A theory for multiresolution signal decomposition: the wavelet representation", IEEE transactions on pattern analysis and machine intelligence, vol. 11, no. 7, (1989), pp.674-693.

[12] F. Strasser, M. Muma and A.M. Zoubir, "Motion artifact removal in ECG signals using multi-resolution thresholding", In Signal Processing Conference (EUSIPCO), 2012 Proceedings of the 20th European, IEEE, (2012), pp. 899-903.

[13] C.H. Chang, H.J. Ko and K.M. Chang, "Cancellation of high-frequency noise in ECG signals using adaptive filter without external reference", In Biomedical Engineering and Informatics (BMEI), 2010 3rd International Conference on, IEEE, vol. 2, (2010), pp. 787-790.

[14] A.B. Sankar, D. Kumar and K. Seethalakshmi, "Performance study of various adaptive filter algorithms for noise cancellation in respiratory signals", Signal processing: An international journal (SPIJ), vol. 4, no. 5, (2010). p. 267. 